\title{
Dewetting dynamics of helium films on cesiated surfaces
}

\author{
Dietmar Reinelt, Andreas Engel, Jürgen Klier* \\ Fakultät für Physik, Universilät Konstanz, 78457 Konstanz, Germany
}

\begin{abstract}
The nonwetting of liquid ${ }^{4} \mathrm{He}$ on $\mathrm{Cs}$ below $\approx 2 \mathrm{~K}$ is now well established. However, there exist discrepancies between the measured contact angles and hysteresis at the phase transition of various groups. These are attributed to the influence of the underlying Cs surface. Little is known, however, about the morphology of this surface. There is also a lack of information concerning the dewetting dynamics of metastable ${ }^{4} \mathrm{He}$ films and the dewetting process of ${ }^{4} \mathrm{He}$ drops on this weak binding surface Cs. We present results showing the influence of surface properties on the dewetting behaviour of these metastable films and discuss the scenario of dewetting of a macroscopic helium drop.
\end{abstract}

Keywords: Cesium; ${ }^{4} \mathrm{He}$ film; Surface morphology; Wetting

Liquid ${ }^{4} \mathrm{He}$ is extremely weakly bound to $\mathrm{Cs}$ and hence shows nonwetling below $T_{w} \approx 2 \mathrm{~K}[1]$ (see Ref. [2] for the first experimental evidence for nonwetting of ${ }^{4} \mathrm{He}$ on Cs). At $T \ll T_{w}$ only a microscopically thin ${ }^{4} \mathrm{He}$ film exists on $\mathrm{Cs}$ forming a two-dimensional gas [3]. When cooling a Cs surface below $T_{w}$, which is initially covered with a thick ${ }^{4} \mathrm{He}$ film and in contact with a ${ }^{4} \mathrm{He}$ reservoir, then in most cases this film does not dewet, but rather a metastable thick film remains. However, most experiments show strikingly different hysteresis in film thickness and contact angle [4-6]. This hysteretic wetting behaviour is attributed to the surface properties of the underlying substrate, namely roughness and chemical impurities.

We have studied the dynamics of dewetting ${ }^{4} \mathrm{He}$ films on several Cs substrates imaged by a surface plasmon microscope [4]. Our Cs substrates are $\approx 15 \mathrm{ML}$ thick films evaporated at low $\mathrm{T}$ onto thin $\mathrm{Ag}$ films. We analysed the profile of a contact line separating a metastable ${ }^{4} \mathrm{He}$ film on Cs from a dry Cs area. This gives information about the stability of the film and its dependence on the surface morphology. We also studied the dynamics of isolated superfluid ${ }^{4} \mathrm{He}$ drops on an otherwise dry $\mathrm{Cs}$ surface. To characterize the Cs surface due to chemical

\footnotetext{
* Corresponding author.

E-mail address: juergen.klicr@uni-konstanz.dc (J. Klier)
}

impurties, we have done local measurements of the work function, $\Phi(v)$, i.e. measured the current of the photoelectrons from our $C$ s surface as a function of the cut-oft light wavelength. A detailed description of the setup is given in Rer. [7].

Fig. 1 shows the dewetling of an isolated ${ }^{4} \mathrm{He}$ drop on $C_{5}$ at $T<T_{w}$. The $C s$ surface is in thermodynamical equilibrium, i.e. there is no thick melastable film on the surface. The dewetting is only possible via the gas phase and the thin nonwetting film surrounding it. If this film is in its $2 \mathrm{D}$-gas phase then mass transport is negligible and the main mechanism has to be evaporation from the drop. However, if the thin film is in a $2 \mathrm{D}$-liquid state [8] transport through it could play a role in the dynamics of the dewelting process. Due to our limiting resolution for the thin film we must await further work to answer these questions.

In close vicinty to this drop is a contact line, see Fig. la, separating the nonwet Cs from a $C s$ area which is permanently covered with a thick superfluid ${ }^{4} \mathrm{He}$ film after first deposition of a helium drop on this area. When further drops are deposited on this part of the Cs the dewetting process is much faster than on the dry Cs part. The dewetting time is always $<3 \mathrm{~s}$, although these deposited drops are much bigger in size than the isolated ones. The transport of helium out of these big drops is dominated through the already existing superfluid metastable film which is in contact to the surrounding wetted 

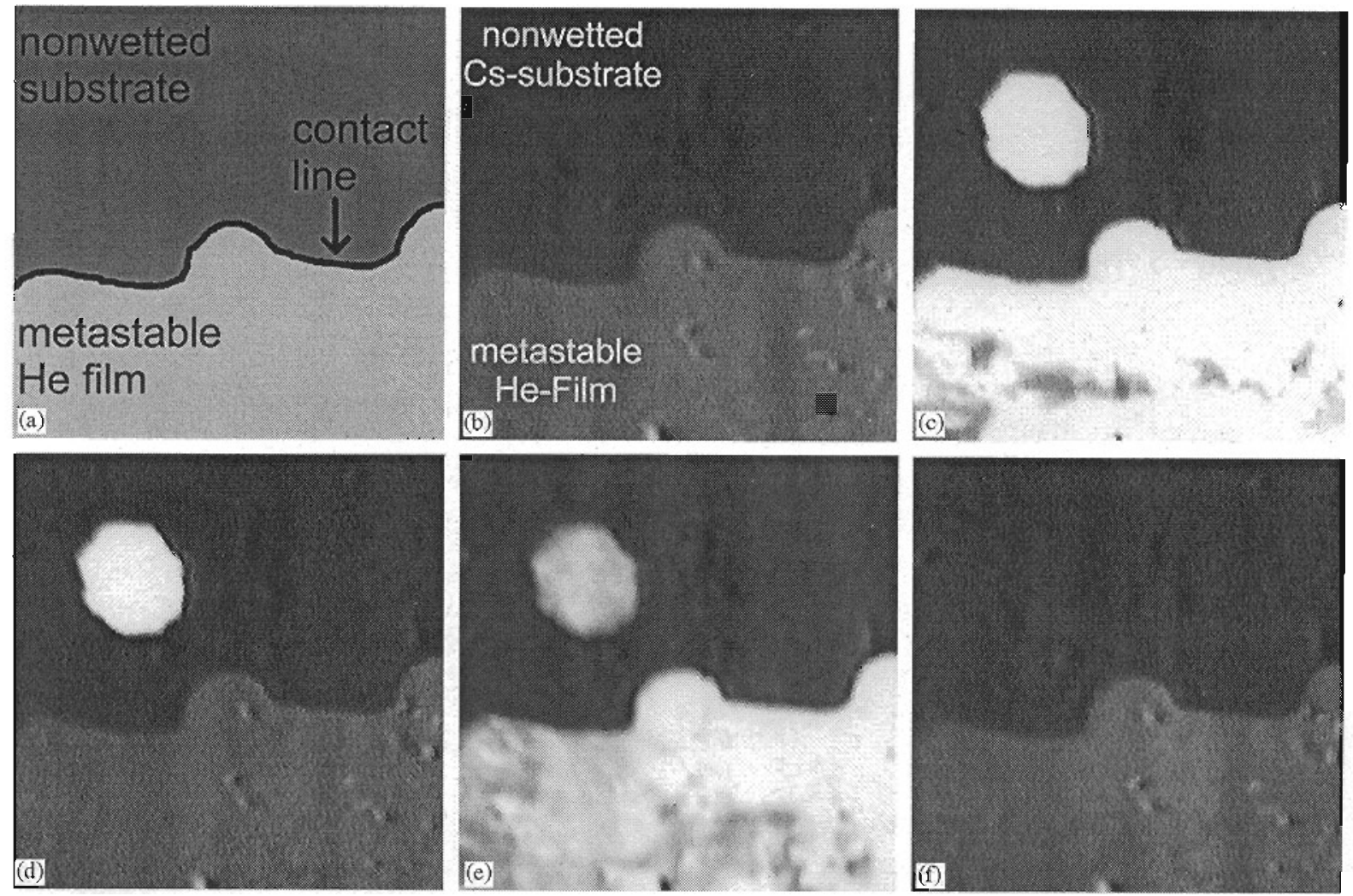

Fig. I. Dewerting of ${ }^{4} \mathrm{He}$ drops deposited on a horizontal Cs surrace $\left(4 \times 4 \mathrm{~mm}^{2}\right)$ at $T=1.38 \mathrm{~K}$, shown via a surface plasmon nicroscope. The upper part of each picture (c-e) shows the dewetting of an isolated drop on the dry Cs-part, deposited at $t=0 \mathrm{~s}$ with a dewetting time of $\approx 8.1 \mathrm{~s}$. During this time several big drops were deposited on the lower part of the $\mathrm{Cs}$ ( $\mathrm{c}$ and $\mathrm{e}$ ), which is always covered with a metastable ${ }^{4} \mathrm{He}$ film and separated from the dry part via a sharp fixed contact line. Picture fis identical to $b$, i.e. no thick ${ }^{4} \mathrm{He}$ film is left on the upper part. The grey area indicates a thick metastable film, the black area the dry Cs. (b) $\iota=-0.3 \mathrm{~s} ;$ (c) $\iota=1.3 \mathrm{~s}$; (d) $t=4.8 \mathrm{~s} ;(e) t=8.0 \mathrm{~s} ;$ (f) $t=11.8 \mathrm{~s}$.

Ag area (not shown). The contact line to the dry Cs is fixed although during deposition of the big drops bulk material moves up to this line. The fraying of this line indicates a rough Cs surface with many pinning centres.

To check on the influence of chemical impurities on the wetting properties, we have scanned our Cs surface and measured the current of the photoelectrons (every 0.5 mm over an area of $\approx 0.2 \mathrm{~mm}^{2}$ ). For pure $\operatorname{Cs} \phi=1.8 \mathrm{eV}$ [9]. Our Cs surface showed variations in $\Phi(r)$ between 1.7 and $1.9 \mathrm{eV}$ over the whole area. However, there was no correlation between the observed wetting properties and $\Phi(r)$. So chemical impurities can be excluded as the reason of the metastable film and the sharp contact line. A possible explanation is that the wetted parts are very rough which enhances wetting.

In conclusion we observed high stability of metastable ${ }^{4} \mathrm{He}$ films on some Cs surfaces. Other Cs surfaces, however, show sponlaneous disappearance of this film below $T_{w}$ which infuences the dynamics of dewetting helium drops. Our results show that the dynamics of ${ }^{4} \mathrm{He}$ films on cesiated surfaces are strongly dependent on the film quality. As a consequence these effects should also have an influence on the interface lree energics of this system, important for wetting parameters like the contact angle [10]. Characterization of the Cs surface is essential for furthet wetting studies on this otherwise ideal system. In addition, measuring the film thickness of the equilibrium 'thin film' seems essential to understand the wetting properties.

\section{Acknowledgements}

We thank P. Leiderer for useful discussions and the DFG-Schwerpunkt 'Benelzung' for support.

\section{References}

[1] E. Cheng et al, Phys. Rev. Lett. 67 (1991) 1007.

[2] P.J. Nacher, J. Dupont-Roc, Phys, Rev. Leti. 6 ? (1991) 2966. 
[3] P. Stefanyi, J. Klier, A.F.G. Wyat, Phys. Rev. Letl. 73 (1994) 692.

[4] J. Klier et al., Phys. Rev, Lett. 75 (1995) 3709

[5] E. Rolley, C. Guthmann, J, Low Temp. Phys. 108 (1997) 1.

[6] D. Ross et al., J. Low Temp. Phys. 111 (1998) 1.

[7] D. Reinelt, J. Klier, P. Leiderer, J. Low Temp. Phys. 113 (1998) 805 .
[8] J. Klier, A.F.G. Wyatt, J. Low Tcinp. Phys. 113 (1998) 817

[9] C.C. Addison, Chemistry of Alkali Metals, Wiley, New York, 1985.

[10] J. Klier, A F.G. Wyatt, in these Proceedings (LT.22) Physica B 284-288 (2000). 